\title{
Knowledge and Attitude of Men Towards Participation in Their Wives' Perinatal Care
}

\author{
Farzaneh Soltani ${ }^{1}$, Manizheh Majidi ${ }^{2}$, Fatemeh Shobeiri ${ }^{1}$, Parisa Parsa ${ }^{3 *}$, Ghodratollah Roshanaei ${ }^{4}$
}

\begin{abstract}
Objectives: Improving the maternal health requires an understanding of the men's level of knowledge as well as the attitude about participation in their wives' perinatal care in different societies. The present study aims to investigate men's knowledge and attitude about participation in their nulliparous wives' perinatal care.

Materials and Methods: In this descriptive cross-sectional study, 300 husbands of nulliparous women completed the questionnaire of "men's knowledge and attitude about participation in prenatal care", in a referral perinatal care clinic in Hamadan, Iran, in 2015. Results: The level of knowledge about the wives' perinatal care in more than half of the men (58\%) was poor and in nearly half of them (41.7\%) was moderate. Based on different aspects of the perinatal care, the knowledge level of 59.7\%, 69.7\%, 52.3\%, 63.3\%, $64.7 \%, 66.7 \%, 51.7 \%, 62 \%, 84 \%$, and $78.7 \%$ of the men was poor in the physical changes, general health, nutrition, exercise, sexual health, risk signs, mental and psychological changes in pregnancy, delivery, puerperium, and neonatal care, respectively. None of the men had good knowledge about the aspects related to postpartum care (including puerperium and neonatal care). Further, the majority of men $(65.3 \%)$ had a positive attitude towards participation in perinatal care.

Conclusions: In the present study, the emphasis was put on the need for training the men interested in participating in various perinatal cares, especially physical changes during pregnancy, prenatal nutrition, risk signs during pregnancy, and maternal as well as neonatal postpartum cares.

Keywords: Perinatal care, Men, Participation, Knowledge, Attitude
\end{abstract}

\section{Introduction}

Pregnancy is an important, critical, and meanwhile glorious period in any woman's life. The physical, psychological, and mental health of the woman in this period significantly affects the fetal health, success in normal delivery, and breastfeeding (1). One of the important strategies for improving the physical, mental, and psychological health of women during pregnancy is the participation and support of their relatives, especially the husbands, in this momentous issue (2).

For most of the men, the first experience of "becoming a father" is associated with significant changes in the personal identity and relationship with their wives (3, 4); Moreover, most women expect their husbands to pay attention to their health (5). In fact, male participation in the prepartum, and postpartum care is requested by women (6) and the evidence shows that both women and men consider male participation in prenatal care necessary (7).

The role of men in pregnancy issues is one of the challenging subjects. Some researchers believe that pregnancy and delivery are women-specific experiences and deliberately consider men's role as a secondary one.
From some other researchers' perspective, attempting to involve men in the affairs related to women's health not only strengthens the relationships within the family but also influences the quality of the relationship between women and men in the society (8). Some other researchers believe that engagement in the prenatal care provides good opportunities for fathers to play an important and positive role in improving the health of their wives and infants and get prepared for adapting to their new role as the parent (9).

Regardless of different views, the World Health Organization (WHO) that has given the top priority to the reduction of mothers' mortality due to pregnancy and parturition in its Reproductive Health Program believes that men are good partners for providing mothers' health (10). In 2009, United Nations Population Fund announced that despite substantial evidence on the advantages of male participation, such participation during pregnancy, at the time of delivery, and in neonatal care has not been yet effectively promoted at the global level (11). On this basis, the slogan of the World Population Day in 2010 was announced to be "Male participation in improving maternal health", and it was emphasized that fathers 
should participate in all the family-related programs, including prepartum, and postpartum care and should be encouraged to support the safe motherhood program (12).

Male participation in prenatal and delivery cares leads to positive outcomes $(13,14)$. It has been shown that such participation can reduce the risk of preterm birth with low birth weight as well as intrauterine growth restriction and neonatal mortality (15). Further, there is a significant and direct relationship between wives' mental health and male participation during pregnancy (16). The physiological and epidemiological evidence indicates that male participation in prenatal care leads to the reduction of stress (17), acceptance of prenatal care by pregnant women $(18,19)$ and acceptance of the parental roles at the early stages of pregnancy $(20,21)$. In fact, men play a special role in improving the maternal health (22) and through participation in the safe motherhood programs, they could protect women's health and life in different ways. Protecting mother's health during pregnancy, providing the ground for the presence of a skilled and trained person at the time of delivery, helping mothers after delivery, and undertaking the role of a responsible father in the family are some examples of the men's active participation in the family (23). Furthermore, father's participation in prenatal care and his presence during delivery lead to a stronger relationship between the father and infant at the following stages of the infant's development and would be associated with positive outcomes related to cognitive, developmental, and social behaviors of the infant (24). In the study by Mortazavi and Mirzaii, better understanding of the delivery-related problems, better understanding of pregnancy events, more protection of wives, reduction of men's worries, better relationship of the spouse with fetus, and reduction of the labor pain were mentioned by the pregnant women as the positive consequences of male participation in the prenatal care (25). Moreover, male participation in the maternal and neonatal cares provides new opportunities for health caregivers to train the future fathers in order to accompany them in providing their family's health (9).

However, for any reason, men are on the margin of the services provided for mothers (22). Nevertheless, men have given positive responses to the efforts made for achieving their participation during pregnancy and delivery (11). For example, in the study by Carter in Guatemala, $90 \%$ of the fathers participated in the prenatal or delivery cares or in the neonatal care program (9). Further, David and Kentenich found that more than 70\% of the men participating in the labor tended to support their wives even during difficult deliveries and surgical interventions (26).

While male participation in women's perinatal cares shows an increase in developed countries $(3,27)$, in other regions of the world, men's norms in playing their role in maternal and neonatal cares are changing (9). These norms are mostly rooted in the cultural and social backgrounds of different societies and need to be accurately known. Promoting male participation as an important strategy to achieve the goals of the third millennium development, including enabling women and improving maternal health (6), requires recognition of men's level of knowledge and attitude about participation in their wives' perinatal cares in different societies, and such a goal is of greater importance in a country like Iran with young population and strong traditional and cultural roots. Therefore, the present study aims to investigate men's knowledge and attitude about participation in the primigravida wives' perinatal cares.

\section{Materials and Methods}

This descriptive cross-sectional study was conducted on 300 husbands of the nulliparous women referring to Prenatal Clinic of Fatemieh hospital in Hamadan in 2015. The sample consisted of the husbands of the healthy nulliparous pregnant women with a normal pregnancy and gestational age of less than 32 weeks, without using any assisted reproductive technology (ART).

The participants were selected according to the inclusion criteria and through simple random sampling method. After convincing the couples to participate in the study and obtaining their informed consent by the researcher, the questionnaires were completed by them. The statistical analyses were performed using SPSS statistical software (version 19.0), and $P<0.05$ was considered statistically significant.

\section{Measures}

1. "Men's knowledge and attitudes about participation in perinatal care" questionnaire: The questionnaire was designed by the research team using a questionnaire designed by Simbar et al (7) and based on the contents of a booklet entitled "Prenatal Training and Preparation for Delivery" (Ministry of Health and Medical Education, Iran). The "knowledge" part of the questionnaire consisted of 70 questions and 12 aspects of perinatal care including physical changes during pregnancy, common prenatal complications, nutrition, general health, prenatal exercises, sexual health, risk signs during pregnancy, parents' mental changes during pregnancy, delivery, puerperium, breastfeeding, and neonatal care. Positive and zero points would be given to the correct and incorrect responses to each question, respectively. By leveling the obtained scores (both total score and the scores related to various aspects of perinatal care), the scores between 0 and 33.3, between 33.3 and 66.6, and between 66.6 and 100 were considered poor, medium, and good levels of knowledge, respectively. The "attitude" part of the questionnaire consisted of 15 items with a 3-point Likert scale, including disagree, neutral, and agree, in which the scores of 1, 0 , and 2 were considered for disagree, neutral, and agree, respectively. Then the total scores were calculated. The negative, 
neutral, and positive attitudes were attributed to the scores between 0 and 33, between 34 and 67, and between 68 and 100, respectively. The validity of the questionnaire was assessed using different experts' opinions, and its reliability was calculated using Cronbach a coefficient (0.89).

2. "Demographic and obstetric characteristics" questionnaire included age, family size, education, ethnicity, occupation, housing status and monthly income of the couples, marriage duration, history of infertility, number of abortions, number of deceased neonates, date of last menstruation, gestational age, and estimated date of delivery.

\section{Results}

The mean age of the women and men was $25.81 \pm 4.619$ and 30.09 \pm 4.231 years old, respectively, and the mean marriage duration was $3.73 \pm 2.016$ years. The majority of the couples $(84 \%)$ were living together, not with their relatives. The mean gestational age of the women was $25.89 \pm 4.013$ weeks, and $97.7 \%$ of whom were receiving prenatal care. In addition, $48 \%$ of the couples were living in rental houses. Table 1 shows other demographic characteristics of the couples.

In general, the level of knowledge about perinatal care was poor in more than half of the men (58\%) and medium in nearly half of them (41.7\%). Only one of them had a good level of knowledge. In terms of different aspects of perinatal care, the knowledge level of $59.7 \%, 69.7 \%, 52.3$ $\%, 63.3 \%, 64.7 \%, 66.7 \%, 51.7 \%, 62 \%, 84 \%$, and $78.7 \%$ of the men was poor in the fields of physical changes, public health, nutrition, exercise, sexual health, risk signs, mental and psychological changes during pregnancy, delivery, puerperium, and neonatal care, respectively. Majority of the men $(42.7 \%)$ had a medium level of knowledge in the breastfeeding axis. None of the men had a good level of knowledge about postpartum cares-related aspects (including puerperium and neonatal care); furthermore, only $2.7 \%$ of the men had a good level of knowledge about the physical changes during pregnancy (Table 2).

The majority of the men $(65.3 \%)$ had a positive attitude towards their participation in the perinatal care while the attitude of $34 \%$ of them was neutral. There was only one man with a negative attitude towards participation in perinatal care. Table 3 shows the distribution of the samples according to their attitude towards participation in perinatal care. The highest percentage of the men's agreement (98.5\%) was related to the statement "pregnant women need more mental support during pregnancy" and the statement "I help my wife because I believe that the newborn child belongs to both of us" was the next in ranking. In contrast, the highest percentage of the men's disagreement $(81.7 \%)$ was related to the statement " $I$ would not help during pregnancy because I think these tasks are related only to mothers".

According to Table 4, men's knowledge and attitudes about participation in perinatal care was not related to their age, occupation, ethnicity, marriage duration, gestational age, history of abortion, and stillbirth; however, it had a significant relationship with the male education $(P=0.005)$, and socioeconomic levels of the couples including family size $(P=0.000)$ and housing status $(P=0.001)$.

\section{Discussion}

Participation of men in their wives' perinatal care is currently considered as an essential element of WHO's initiative for safe pregnancy (12). Such participation is of special importance in developing countries, in which men are often the policy- and decision-makers in the family and at the society level (10). International studies have demonstrated that men usually respond to the participation request as "We will participate", but their poor performance is due to the lack of knowledge or very low level of knowledge about various needs of the pregnant women and their own roles and responsibilities (22).

Table 1. Demographic Characteristics of Male and Female Participants

\begin{tabular}{|c|c|c|c|c|c|c|}
\hline \multirow{2}{*}{ Variable } & \multicolumn{2}{|c|}{ Females $(n=300)$} & \multicolumn{2}{|c|}{ Males $(n=300)$} & \multicolumn{2}{|c|}{ Total $(\mathrm{N}=600)$} \\
\hline & No. & $\%$ & No. & $\%$ & No. & $\%$ \\
\hline \multicolumn{7}{|l|}{ Education } \\
\hline Primary school & 8 & 2.7 & 14 & 4.7 & 22 & 7.4 \\
\hline Middle school & 63 & 21.0 & 59 & 19.7 & 122 & 40.7 \\
\hline High school & 96 & 32.0 & 106 & 35.3 & 202 & 67.3 \\
\hline University & 132 & 44.0 & 120 & 40.0 & 252 & 84 \\
\hline \multicolumn{7}{|l|}{ Ethnicity } \\
\hline Fars & 162 & 54.0 & 158 & 52.7 & 320 & 106.7 \\
\hline Kurd & 33 & 11.0 & 29 & 9.7 & 62 & 20.7 \\
\hline Lor & 14 & 4.7 & 15 & 5.0 & 29 & 9.7 \\
\hline Turk & 89 & 29.7 & 95 & 31.7 & 184 & 61.4 \\
\hline \multicolumn{7}{|l|}{ Occupation } \\
\hline Unemployed/householder & 272 & 90.7 & 2 & 0.7 & 274 & 91.4 \\
\hline Worker & - & - & 44 & 14.7 & 44 & 14.7 \\
\hline Office personnel & 13 & 4.3 & 89 & 29.7 & 102 & 34 \\
\hline Others & 11 & 3.7 & 162 & 54.0 & 173 & 57.7 \\
\hline
\end{tabular}


Table 2. Men's Levels of Knowledge According to Different aspects of Perinatal Care

\begin{tabular}{|c|c|c|c|c|c|c|}
\hline \multirow{3}{*}{ Perinatal Care Axes } & \multicolumn{6}{|c|}{ Knowledge Level } \\
\hline & \multicolumn{2}{|c|}{ Good } & \multicolumn{2}{|c|}{ Average } & \multicolumn{2}{|c|}{ Poor } \\
\hline & No. & $\%$ & No. & $\%$ & No. & $\%$ \\
\hline Physical changes & 8 & 2.7 & 113 & 37.7 & 179 & 59.7 \\
\hline Common Complications & 10 & 3.3 & 146 & 48.7 & 144 & 48.0 \\
\hline Nutrition & 17 & 5.7 & 126 & 42.0 & 157 & 52.3 \\
\hline General health & 20 & 6.7 & 71 & 23.7 & 209 & 69.7 \\
\hline Exercising & 17 & 5.7 & 93 & 31.0 & 190 & 63.3 \\
\hline Sexual health & 19 & 6.3 & 87 & 29.0 & 194 & 64.7 \\
\hline Risk signs & 17 & 5.7 & 83 & 27.7 & 200 & 66.7 \\
\hline Psychological changes & 57 & 19.0 & 88 & 29.3 & 155 & 51.7 \\
\hline Delivery & 114 & 38.0 & - & - & 186 & 62.0 \\
\hline Puerperium & - & - & 48 & 16.0 & 252 & 34.0 \\
\hline Breast feeding & 71 & 23.7 & 128 & 42.7 & 101 & 33.7 \\
\hline Neonatal care & - & - & 64 & 21.3 & 236 & 78.7 \\
\hline Total knowledge & 1 & 0.3 & 125 & 41.7 & 174 & 58.0 \\
\hline
\end{tabular}

Table 3. Distribution of Male Participants According to Their Attitude Towards Male Participation in Perinatal Care

\begin{tabular}{|c|c|c|c|c|c|c|}
\hline \multirow{3}{*}{ Statement } & \multicolumn{6}{|c|}{ Attitude } \\
\hline & \multicolumn{2}{|c|}{ Agree } & \multicolumn{2}{|c|}{ Neutral } & \multicolumn{2}{|c|}{ Disagree } \\
\hline & No. & $\%$ & No. & $\%$ & No. & $\%$ \\
\hline Education on prenatal care is necessary for mothers & 292 & 97.3 & 7 & 2.3 & 1 & 0.3 \\
\hline Education on prenatal care is necessary for fathers & 237 & 79.0 & 47 & 15.7 & 15 & 5.0 \\
\hline Pregnant mothers need a lot of emotional support during pregnancy & 294 & 98.5 & 4 & 1.3 & 2 & 0.7 \\
\hline I help to my wife because the child belongs to us both & 293 & 97.7 & 6 & 2.0 & 1 & 0.3 \\
\hline I like to accompany her in the prenatal visits & 239 & 76.7 & 50 & 16.7 & 11 & 3.7 \\
\hline Women raised children without help in the past & 149 & 49.7 & 87 & 29.0 & 64 & 21.3 \\
\hline Wives have no job except household and taking care of her self & 148 & 49.3 & 59 & 19.7 & 91 & 30.3 \\
\hline The attendance of mother lonely enough in readiness classes of before delivery & 115 & 38.3 & 70 & 23.3 & 114 & 38.0 \\
\hline The father should participate in readiness classes of before delivery & 179 & 59.7 & 81 & 27.0 & 40 & 13.3 \\
\hline I would like to be attend during labor pains next to my husband & 234 & 78.5 & 44 & 14.7 & 21 & 7.0 \\
\hline I would like to be attend during delivery next my wife & 235 & 78.3 & 40 & 13.3 & 25 & 8.3 \\
\hline Help to a pregnant mother is not usual in my family & 43 & 14.3 & 16 & 5.3 & 238 & 79.3 \\
\hline Help to a pregnant mother is not usual in our community & 44 & 14.7 & 50 & 16.7 & 203 & 67.7 \\
\hline I do not help in the perinatal period because it is a woman's job & 40 & 13.3 & 14 & 4.7 & 245 & 81.7 \\
\hline Neonatal care is not a men's job & 54 & 18.0 & 26 & 8.7 & 220 & 73.3 \\
\hline
\end{tabular}

Table 4. Correlation Between Obstetrics and Demographic Characteristics of Couples With Men's Knowledge and Attitude Towards Participation in Perinatal Care

\begin{tabular}{|c|c|c|c|c|}
\hline \multirow{2}{*}{ Variable } & \multicolumn{2}{|c|}{ Knowledge } & \multicolumn{2}{|c|}{ Attitude } \\
\hline & Pearson Correlation & $P$ Value & Pearson Correlation & $P$ Value \\
\hline Duration of marriage & 0.026 & 0.147 & 0.086 & 0.660 \\
\hline Gestational age & 0.001 & 0.763 & -0.190 & 0.981 \\
\hline History of abortion & -0.061 & 0.731 & -0.020 & 0.322 \\
\hline History of infant death & 0.034 & 0.394 & -0.050 & 0.560 \\
\hline Male age & 0.88 & 0.173 & 0.079 & 0.131 \\
\hline Family size & -0.069 & 0.000 & -0.222 & 0.244 \\
\hline Male education & 0.272 & 0.005 & 0.163 & 0.000 \\
\hline Male ethnicity & -0.024 & 0.541 & -0.036 & 0.685 \\
\hline Male job & -0.014 & 0.198 & 0.075 & 0.806 \\
\hline Housing status & -0.079 & 0.001 & -0.184 & 0.172 \\
\hline Family income & 0.196 & 0.084 & 0.101 & 0.001 \\
\hline
\end{tabular}


In the present study, almost none of the men had a negative attitude toward participation in their wives' perinatal care. However, the traditional gender roles and social stigma that can have a negative effect on the men's involvement are the major obstacles in this regard (25). For example, in a study by Vermeulen et al, although the men knew that the prenatal cares were important for pregnant women, they often adopted a passive stance in this regard and attributed their inactivity to the external factors (28). In another study by Asefa et al, by expressing their public opinion about the womanliness of the perinatal care, the men referred to their shame for accompanying their wives as the major obstacle to their participation (29).

In the present study, almost all of the men (98.5\%) believed that pregnant women need more mental support during pregnancy. In the study conducted in Tehran, from the perspective of most of men and women, providing mental support for women was the most important concept of participation in perinatal care (7). In the present study, the majority of men (97.3\%) agreed with health and perinatal care training for the future mothers, while $79 \%$ of them agreed with providing such training for the future fathers. In addition, $97.7 \%$ of the men believed that they helped their wives because the newborn baby belonged to both parents, and $73.3 \%$ of them thought of the neonatal cares as a duty for fathers.

Despite the positive attitude of almost all of the men in the present study towards participation, the level of knowledge about the perinatal care was poor in more than half of them and medium in nearly half of them. The men's low level of knowledge about the health behaviors, mental and physical needs of pregnant women, and common complications of pregnancy is considered as an important obstacle to their supportive behaviors, which has also been found in other studies. For example, in the study by Awasthi, only $18.1 \%$ of the men were informed of the delivery complications of their wives (30). Another study conducted in Nigeria indicated that $56.5 \%$ of men had a positive attitude towards maternal health care, while more than half of them had a poor level of knowledge in this regard (31). In another study conducted on 400 men, Olugbenga-Bello et al showed that $42 \%$ of the men had a poor level of knowledge, while more than half of them (51.2\%) had a good attitude towards maternal health care (31).

In the present study, the knowledge level of the men was investigated based on different issues (including the aspects related to perinatal care). In this regard, only $2.7 \%$ of the men had a good level of knowledge about the physical changes during pregnancy. In the study by Mortazavi et al, the Iranian pregnant women believed that their husbands' lack of knowledge about the prenatal changes and its complications is one of the important causes of the men's non-participation, and their main suggestion in this regard was holding some training sessions for men and their gradual preparation. Studies performed in other countries have also found that one of the main causes of the men's non-participation in maternal cares is their lack of knowledge and unawareness of the pregnancy complications, and improvement of male participation could be achieved by increasing and improving their knowledge of the prenatal cares (29). Further, the study by Iliyasu et al indicated that the men had a very poor level of knowledge about the skills of partnership during delivery (6.2\%), rescuing mother in emergency cases (19.5\%), or transferring mother at the onset of labor pain (24.2\%) (32).

In the current study, only $5.7 \%$ of the men had a good knowledge of prenatal nutrition and risk signs during pregnancy, and none of them had good level of knowledge of the postpartum cares-related aspects (including puerperium and neonatal cares). This is while informing and preparing the men for facing the problems during pregnancy and early detection of the maternal risk signs can reduce the delays in helping mothers and, as a result, reduce the maternal morbidity and mortality (33). This becomes especially important when we know that $99 \%$ of the maternal deaths occur in developing countries, where men are the principal decision-makers on the women's access to the health services and affect the health consequences (33). In this regard, more than $95 \%$ of the men participating in one study in Iran had mentioned knowing the risk signs during pregnancy, postpartum problems, and prenatal nutrition, respectively, as the educational content required by men (7).

Among various aspects of perinatal care, the highest number of the men enjoying a good level of knowledge was related to the field of delivery and, then, breastfeeding. Although the male participati o $\mathrm{n}$ during pregnancy and after delivery seemed to be more useful than their participation at the time of delivery $(33,34)$, some other studies have also shown that men would probably have a tendency to participate in the issues in which there would be a greater need for their intervention and assistance (7). Extensive and comprehensive in formation and training on the benefits of breastfeeding provided by the Iranian healthcare and treatment system might have a considerable and undeniable effect on the level of knowledge of the future fathers in this regard.

In our study, the men's level of knowledge and attitude about participation in their $\mathrm{w}$ ives' perinatal care was not related to the obstetric factors such as history of abortion and stillbirth, which might be due to the low incidence of the history of abortion and stillbirth among the participants; however, the ir level of knowledge and attitude was significantly related to socioeconomic factors, including education, family size and housing status. In the study by Rabieipoor et al in Iran, there was no significant relationship between the male participation and any of the demographic characteristics (16); however, in the study by Simbar et al, the attitude score of the illiterate men was significantly less than that of others (7). In the study by Martin et al, fathers with the education level of lower than 
high school had less rate of participation during pregnancy (18). Another important point is that the mean age of the men participating in our study showed that the majority of them were at fertility ages, indicating the training needs of the young men during the first pregnancy.

In general, the present study and other relevant studies in Iran indicate the presence of a positive attitude among the men towards participation in perinatal care $(7,25)$; thus, it is a great opportunity to take advantage of the presence of men as important partners for improving the mothers' health and reducing their mortality (9). The health of the mother and infant during pregnancy, delivery, and after delivery requires cooperation between the couples, and fathers can learn such participation and cooperation through training (35). In addition, similar to the mother, the father should undergo some changes in order to adapt to his new role; however, it is sometimes more difficult for fathers because they are ignored by both health caregivers and others (36).

Numerous studies in various countries have revealed the need for training with the aim of increasing fathers' participation in perinatal care and its positive effect on the maternal and neonatal health. The importance of the trained men is more emphasized when we know that training men has led to positive outcomes, including establishment of stronger relationships with wives (3), earlier initiation of prenatal care (18), reinforcement of morale and parents' sense of belonging to their children (37), adoption and continuation of appropriate health behaviors and discontinuation of unhealthy behaviors such as smoking during pregnancy (18), achieving better understanding of delivery-related problems and pregnancy complications by men, reduction of men's worries, reduction of labor pain, neonatal care (9), initiation and continuation of breastfeeding (38), family planning $(4,39)$, intelligent actions in case of delivery complications (33) in various studies.

This study, which is one of the few studies that have addressed the male participation in perinatal care, focuses only on men's knowledge and attitude about participation in their wives' perinatal care, and their performance in this regard has not been considered. It should be noted that male participation in maternal cares is a process of social and behavioral change that must be created in men so that they could undertake more responsible roles in maternal healthcare and ensure the health of mothers and infants (40).

\section{Conclusions}

The present study emphasizes the importance of training the men interested in participating in different perinatal cares, especially physical changes during pregnancy, prenatal nutrition, risk signs during pregnancy, and maternal as well as neonatal postpartum cares. It is necessary to conduct more extensive studies to identify the social and cultural factors forming the behaviors and performance of men in terms of their participation and provide the cultural ground for developing the social and behavioral changes.

\section{Conflict of Interests}

Authors declare that they have no conflict of interests.

\section{Ethical Issues}

The research was approved by Research Deputy of Hamadan University of Medical Sciences [IR.UMSHA. REC.1394.254]. All participants were asked to sign an informed written consent before the start of data collection.

\section{Financial Support}

Research Deputy of Hamadan University of Medical Sciences, Hamadan, Iran.

\section{Acknowledgements}

The authors sincerely appreciated Research Deputy of Hamadan University of Medical Sciences for the financial support. In addition, all participants and personnel in Prenatal Clinic of Fatemieh hospital in Hamadan who helped in the research process are appreciated.

\section{References}

1. Burroughs A, Leifer G. Maternity nursing: an introductory text. Saunders; 2001.

2. Leifer G. Maternity Nursing. Elsevier Saunders; 2007.

3. Diemer GA. Expectant fathers: influence of perinatal education on stress, coping, and spousal relations. Res Nurs Health. 1997;20(4):281-293. doi:10.1002/(SICI)1098240X(199708)20:4<281::AID-NUR2>3.0.CO;2-C

4. Turan JM, Nalbant H, Bulut A, Sahip Y. Including expectant fathers in antenatal education programmes in Istanbul, Turkey. Reprod Health Matters. 2001;9(18):114-125.

5. Dragonas TG. Greek fathers' participation in labour and care of the infant. Scand J Caring Sci. 1992;6(3):151-159. doi:10.1111/j.1471-6712.1992.tb00143.x

6. Greene ME, Mehta M, Pulerwitz J, Wulf D, Bankole A, Singh S. Involving men in reproductive health: Contributions to development. Washington, DC: Millennium Project; 2006.

7. Simbar M, Nahidi F, Tehrani FR, Ramezankhani A. Fathers' educational needs for perinatal care in urban Iran: a qualitative approach. J Biosoc Sci. 2010;42(5):633-641. doi:10.1017/s0021932010000167

8. Carter M. Husbands and maternal health matters in rural Guatemala: wives' reports on their spouses' involvement in pregnancy and birth. Soc Sci Med. 2002;55(3):437-450.

9. Carter MW, Speizer I. Salvadoran fathers' attendance at prenatal care, delivery, and postpartum care. Rev Panam Salud Publica. 2005;18(3):149-156.

10. World Health Organization. Strategy to accelerate progress towards the attainment of international development goals and targets related to reproductive health. Reprod Health Matters. 2005;13(25):11-18.

11. UNPF. Enhancing Mens Roles and Responsibilities in Family Life. A New Role for Men. Available from: URL: http://www.unfpa.org/intercenter/role4men/enhancin 
html.

12. UNPF. Male Involvement in Maternal Health Critical to Saving Women's Lives, Say UN Leaders. United Nations Population Fund; 2007. https://www.unfpa.org/fr/ node/6444.

13. Alio AP, Kornosky JL, Mbah AK, Marty PJ, Salihu HM. The impact of paternal involvement on feto-infant morbidity among Whites, Blacks and Hispanics. Matern Child Health J. 2010;14(5):735-741. doi:10.1007/s10995-009-0482-1

14. Alio AP, Bond MJ, Padilla YC, Heidelbaugh JJ, Lu M, Parker WJ. Addressing policy barriers to paternal involvement during pregnancy. Matern Child Health J. 2011;15(4):425430. doi:10.1007/s10995-011-0781-1

15. Feldman PJ, Dunkel-Schetter C, Sandman CA, Wadhwa PD. Maternal social support predicts birth weight and fetal growth in human pregnancy. Psychosom Med. 2000;62(5):715-725.

16. Rabieipoor S, Khodaei A, Radfar M, khalkhali H. The relationship between husbands' participation in prenatal care and mental health of pregnant women referred to health centers in Urmia, 1392. J Urmia Nurs Midwifery Fac. 2015;13(4):338-347.

17. Ghosh JK, Wilhelm MH, Dunkel-Schetter C, Lombardi CA, Ritz BR. Paternal support and preterm birth, and the moderation of effects of chronic stress: a study in Los Angeles county mothers. Arch Womens Ment Health. 2010;13(4):327-338. doi:10.1007/s00737-009-0135-9

18. Martin LT, McNamara MJ, Milot AS, Halle T, Hair EC. The effects of father involvement during pregnancy on receipt of prenatal care and maternal smoking. Matern Child Health J. 2007;11(6):595-602. doi:10.1007/s10995-007-0209-0

19. Zambrana RE, Dunkel-Schetter C, Scrimshaw S. Factors which influence use of prenatal care in low-income racialethnic women in Los Angeles County. J Community Health. 1991;16(5):283-295.

20. Dolan A, Coe C. Men, masculine identities and childbirth. Sociol Health Illn. 2011;33(7):1019-1034. doi:10.1111/ j.1467-9566.2011.01349.x

21. Gungor I, Beji NK. Effects of fathers' attendance to labor and delivery on the experience of childbirth in Turkey. West J Nurs Res. 2007;29(2):213-231. doi:10.1177/0193945906292538

22. World Health Organization. Programming for male involvement in reproductive health: report of the meeting of WHO regional advisers in reproductive health. Geneva: World Health Organization; 2002.

23. Drennan M. Reproductive health. New perspectives on men's participation. Popul Rep J. 1998(46):1-35.

24. Garfield CF, Isacco A. Fathers and the well-child visit. Pediatrics. 2006;117(4):e637-645. doi:10.1542/peds.20051612

25. Mortazavi F, Mirzaii K. Reason of, barriers to, and outcomes of husbands' involvement in prenatal and intrapartum care program based on midwives' experiences: A qualitative study. Arak Med Univ J. 2012;15(1):104-115.

26. David M, Kentenich H. [Fathers in the labor room--a survey before and after delivery]. Z Geburtshilfe Perinatol. 1993;197(4):195-201.

27. Mullany BC. Barriers to and attitudes towards promoting husbands' involvement in maternal health in Katmandu, Nepal. Soc Sci Med. 2006;62(11):2798-2809. doi:10.1016/j. socscimed.2005.11.013

28. Vermeulen E, Solnes Miltenburg A, Barras J, Maselle $\mathrm{N}$, van Elteren $\mathrm{M}$, van Roosmalen J. Opportunities for male involvement during pregnancy in Magu district, rural Tanzania. BMC Pregnancy Childbirth. 2016;16:66. doi:10.1186/s12884-016-0853-8

29. Asefa F, Geleto A, Dessie Y. Male partners' involvement in maternal ANC care: The view of women attending ANC in Hararipublic health institutions, eastern Ethiopia. Science Journal of Public Health. 2014;2(3):182-188. doi:10.11648/j. sjph.20140203.17

30. Awasthi S, Nandan D, Mehrotra AK, Shankar R. Male participation in maternal care in urban slums of district Agra. Indian J Prev Soc Med. 2008;39(3-4):181-183.

31. Olugbenga-Bello AI, Asekun-Olarinmoye EO, Adewole AO, Adeomi AA, Olarewaju SO. Perception, attitude and involvement of men in maternal health care in a Nigerian community. J Public Health Epidemiol. 2013;5(6):262-270. doi:10.5897/JPHE2013.0505

32. Iliyasu Z, Abubakar IS, Galadanci HS, Aliyu MH. Birth preparedness, complication readiness and fathers' participation in maternity care in a northern Nigerian community. Afr J Reprod Health. 2010;14(1):21-32.

33. Yargawa J, Leonardi-Bee J. Male involvement and maternal health outcomes: systematic review and meta-analysis. J Epidemiol Community Health. 2015;69(6):604-612. doi:10.1136/jech-2014-204784

34. Mortazavi F, Delara M, Akaberi A. Male involvement in prenatal care: impacts on pregnancy and birth outcomes. J Urmia Nurs Midwifery Fac. 2014;12(1):63-71.

35. Alden KR, Lowdermilk DL, Cashion MC, Perry SE. Maternity and women's health care. Elsevier Health Sciences; 2013.

36. McKinney ES, James SR, Murray SS, Nelson K. MaternalChild Nursing. Elsevier Health Sciences; 2013.

37. Mullany BC, Hindin MJ, Becker S. Can women's autonomy impede male involvement in pregnancy health in Katmandu, Nepal? Soc Sci Med. 2005;61(9):1993-2006. doi:10.1016/j.socscimed.2005.04.006

38. Wolfberg AJ, Michels KB, Shields W, O'Campo P, Bronner Y, Bienstock J. Dads as breastfeeding advocates: results from a randomized controlled trial of an educational intervention. Am J Obstet Gynecol. 2004;191(3):708-712. doi:10.1016/j. ajog.2004.05.019

39. Turan JM, Say L. Community-based antenatal education in Istanbul, Turkey: effects on health behaviours. Health Policy Plan. 2003;18(4):391-398.

40. Kululanga LI, Sundby J, Malata A, Chirwa E. Striving to promote male involvement in maternal health care in rural and urban settings in Malawi - a qualitative study. Reprod Health. 2011;8:36. doi:10.1186/1742-4755-8-36

(c) 2018 The Author (s); This is an open-access article distributed under the terms of the Creative Commons Attribution License (http://creativecommons.org/licenses/by/4.0), which permits unrestricted use, distribution, and reproduction in any medium, provided the original work is properly cited. 